\title{
Construção da escala de motivos da procrastinação acadêmica
}

\author{
Gabriela Ballardin Geara \\ Universidade Federal do Rio Grande do Sul, RS, Brasil \\ Nelson Hauck Filho \\ Universidade São Francisco, SP, Brasil \\ Marco Antonio Pereira Teixeira \\ Universidade Federal do Rio Grande do Sul, RS, Brasil
}

\begin{abstract}
Resumo
A procrastinação acadêmica é uma forma de adiamento da realização de tarefas acadêmicas que pode ser prejudicial aos estudantes. Este trabalho busca construir e investigar evidências de validade e fidedignidade da Escala de Motivos da Procrastinação Acadêmica, que avalia a percepção de universitários sobre a frequência de sua procrastinação em tarefas acadêmicas e os motivos associados a ela. Após a construção dos itens e análise semântica por juízes, foi avaliada a relação entre motivos e uma série de variáveis externas, incluindo tarefas mais procrastinadas, conscienciosidade, depressão, impulsividade e gênero. A amostra foi composta por 604 estudantes de graduação e a coleta de dados foi online. Uma análise fatorial exploratória ordinal revelou uma estrutura de dois fatores oblíquos subjacentes aos itens: procrastinação-ansiedade e procrastinação-desmotivação. Apesar do reduzido número de itens, ambos os fatores obtiveram níveis aceitáveis de fidedignidade e apresentaram um padrão similar de correlações com depressão (+) e conscienciosidade (-). Sugestões para estudos futuros são fornecidas.
\end{abstract}

Palavras-chave: motivação; atraso; estudante universitário.

\section{Construction of the academic procrastination scale - reasons}

\begin{abstract}
The academic procrastination is a way of postponing the fulfillment of academic tasks that can be prejudicial to undergraduates. This study aims to elaborate and investigate evidences of validity and reliability of the Academic Procrastination Scale - Reasons, which evaluates the perception of undergraduates about the frequency of their procrastination on academic tasks and the reasons associated with it. The analytical sequence included semantic analysis of the initial item pool, and further external relationships to most procrastinated tasks, conscientiousness, depression, impulsivity, and gender. The sample consisted of 604 undergraduate and data collection was online. Ordinal exploratory factor analysis uncovered a two-dimension latent structure: procrastination-anxiety and procrastination-demotivation. Despite containing few items, the resulting scales yielded acceptable internal consistency, and evidenced a similar pattern of correlations to depression (+) and conscientiousness (-). Suggestions for future studies are provided.
\end{abstract} Keywords: motivation; delay; undergraduate student.

\section{Construción de la escala de razones para procrastinación académica}

\section{Resumen}

La procrastinación académica es una manera de posponer el cumplimiento de tareas académicas que puede ser perjudicial para los estudiantes universitarios. Este estudio tiene como objetivo elaborar e investigar las evidencias de validez y fiabilidad de la Escala de Razones para Procrastinación Académica que evalua de la percepción de los estudiantes acerca de la frecuencia y de las razones de su procrastinación académica. Después de la construcción de los ítems y el análisis semántico por los jueces, se evaluó la relación entre razones y una serie de variables externas, incluidas las tareas más procrastinadas, lo concienzudo, la depresión, la impulsividad y el género. La muestra constenia 604 estudiantes de grado y la recopilación de datos fue online. El análisis factorial exploratorio ordinal descubrió una estructura latente de dos dimensiones: la procrastinación-ansiedad y la procrastinación-desmotivación. A pesar de que contienen pocos itens, las escalas resultantes tienen una consistencia interna aceptable y evidencian un patrón similar de correlaciones con la depresión (+) y la responsabilidad (-). Sugerencias para futuros estudios son fornecidas.

Palabras clave: motivación; dilación; estudiante universitario. 


\section{Introdução}

A procrastinação é definida como o adiamento voluntário de uma atividade pretendida, necessária e/ ou com importância pessoal, apesar de o sujeito esperar potenciais consequências negativas que superariam as consequências positivas desse adiamento (Klingsieck, 2013). Portanto, o indivíduo age por si mesmo sem ter algum motivo de força maior que o impeça de realizar o que pretendia (Klingsieck, 2013).

Em específico, a procrastinação acadêmica é o adiamento de tarefas relacionadas à universidade que não são realizadas dentro do período de tempo esperado ou são deixadas para o último minuto (Schouwenburg, 1995). Nesse contexto, a procrastinação é uma falha no processo de autorregulação da aprendizagem - aquele através do qual o próprio estudante pode controlar e regular seu processo de aprendizagem em termos metacognitivos, motivacionais e comportamentais (Zimmerman, 2001). Dada a importância do tema, este estudo busca apresentar a construção de um instrumento sobre a motivação dos universitários para procrastinar intitulado Escala de Motivos da Procrastinação Acadêmica (EMPA).

Estima-se que a procrastinação acadêmica ocorra com até 95\% dos universitários (O’Brien, 2002), distribuindo-se conforme uma curva normal na população estudantil (McCown, Johnson \& Petzel, 1989). No Brasil, um estudo mostrou que $65 \%$ dos universitários procrastinavam suas atividades acadêmicas ao menos uma vez por semana (Sampaio, 2011). Há evidências também de que cerca de 50\% dos universitários procrastinam de modo problemático, gerando desconforto psicológico e prejuízos acadêmicos (Solomon \& Rothblum, 1984).

Quando um indivíduo procrastina, ele não necessariamente compreende com clareza o que o leva a agir desse modo (Klingsieck, 2013). Além disso, a literatura ainda não oferece conclusões suficientes para o entendimento das origens desse comportamento. Steel (2007) verificou as correlações da procrastinação com 691 variáveis em uma meta-análise. Os resultados revelaram correlações positivas com impulsividade $(\mathrm{Mr}$ $=.41)$ e depressão $(M r=.28)$, bem como correlações negativas com conscienciosidade (organização, autocontrole, motivação para o desempenho; $M r=$ -.62) (Steel, 2007). A procrastinação apresenta uma correlação moderada com a impulsividade (Steel, 2007). Pessoas impulsivas podem ser mais propensas a procrastinar por se envolverem mais com desejos do momento presente, mantendo sua atenção nesse foco. Muitas vezes, os sujeitos mais impulsivos preferem gratificações imediatas, esquecendo ou ignorando as responsabilidades de longo prazo (Steel, 2007).

A procrastinação acadêmica tem sido associada à baixa autoeficácia, baixa autoestima, elevada autoconsciência e elevada autocrítica (Effert \& Ferrari, 1989; Steel, 2007). Possivelmente, procrastinadores são mais emocionais, sobrecarregados e ansiosos, possuem menor necessidade de complexidade cognitiva e tendem a atribuir o sucesso a fatores instáveis e externos (Solomon \& Rothblum, 1984). Os universitários relatam a preguiça como um motivo da procrastinação acadêmica (Grunschel, Patrzek \& Fries, 2013a), sendo que a mesma diferencia-se da procrastinação por se tratar de uma morosidade e aversão à realização de tarefas que exijam esforço físico ou mental (Kerbauy, 2001).

O perfeccionismo tem se destacado como importante fator associado à procrastinação acadêmica (Burka \& Yuen, 1983), sendo reconhecido pelos estudantes entrevistados como um motivo para se procrastinar (Grunschel, Patrzek \& Fries, 2013a). Afinal, ele pode afetar a capacidade de trabalho do indivíduo, paralisando-o quando tenta iniciar ou concluir a tarefa, levando-o à procrastinação (Hollender,1965).

A forma como o estudante percebe algumas características da tarefa (tais como o quanto ela é difícil, complexa, interessante, urgente e demorada) também pode influenciar no quanto ele a procrastina (Grunschel, Patrzek \& Fries, 2013a). Quando o universitário entende que sua tarefa acadêmica é difícil de ser cumprida, ele tende a procrastinar (Pychyl, 2000), sendo relativamente comum os estudantes que procrastinam com frequência apontarem o medo do fracasso como um motivo para seu comportamento (Ozer, Demir, \& Ferrari, 2009).

Sentir-se pressionado a atingir objetivos determinados por si mesmo ou por outros pode ser outra causa da procrastinação acadêmica (Grunschel, Patrzek \& Fries, 2013a). Afinal, alguns estudantes se sentem pressionados pelo objetivo da tarefa em si ou pelas limitações de tempo existentes, de modo que acabam evitando realizá-la. Outros a adiam porque acreditam que trabalham melhor sob pressão (Grunschel, Patrzek \& Fries, 2013a). Por fim, alterações no estado de saúde física (tais como estar doente ou cansado) também foram citadas por universitários como motivos para adiarem a realização de suas tarefas acadêmicas (Grunschel, Patrzek \& Fries, 2013a).

No Brasil, poucos estudos sobre métodos de coleta de dados na avaliação da procrastinação foram localizados. Em um deles, Hamasaki e Kerbauy (2001) investigaram a procrastinação em relação a comportamentos de cuidados com a saúde usando um método qualitativo para compreender as razões e 
os sentimentos relacionados à procrastinação. Outro estudo nacional investigou a procrastinação acadêmica através de um questionário com questões fechadas e abertas que avaliavam frequência, motivos e processo da procrastinação (Sampaio \& Bariani, 2011). Nenhum dos dois estudos, contudo, teve como propósito desenvolver um instrumento de avaliação dos motivos da procrastinação acadêmica. Já em um estudo exploratório, Sampaio (2011) mede a procrastinação acadêmica em relação ao estudo para provas e ao estudo diário, verificando que a procrastinação se mostrou negativamente correlacionada com a autorregulação da aprendizagem.

Em uma busca na literatura sobre o tema, com o descritor "procrastinação", apenas sete trabalhos sobre a procrastinação em contexto de estudo, disponíveis em português, foram localizados nas bases de dados SciELO, BVS, IndexPsi e Banco de Teses da CAPES. Os demais estudos focalizaram a procrastinação a partir de análises da administração ou do campo da saúde. Em termos de escalas de avaliação, apenas a Escala de Procrastinação Acadêmica (Sampaio, 2011) foi localizada. Este instrumento é uma escala de autorrelato, que visa avaliar comportamentos específicos de procrastinação no estudo diário e no estudo para provas em alunos do Ensino Fundamental e Médio. Originalmente, essa escala foi desenvolvida em Portugal e depois validada semântica e culturalmente para o Brasil. Em função disso, pode-se perceber que há escassez de instrumentos que avaliem o comportamento procrastinatório de estudantes universitários brasileiros - o que é um limitador para a realização de mais pesquisas nessa área. Logo, os instrumentos existentes não dão conta de avaliar a percepção dos estudantes universitários brasileiros sobre os motivos que os levam a procrastinar suas tarefas acadêmicas assim como não buscam identificar possíveis relações entre as tarefas acadêmicas mais procrastinadas e os motivos associados a esse comportamento.

O objetivo principal deste estudo foi propor uma escala para avaliar os motivos relacionados à procrastinação acadêmica, pois não foram localizados instrumentos brasileiros sobre esse tema. Assim, os objetivos específicos desse estudo foram: a) construir a Escala de Motivos da Procrastinação Acadêmica para avaliar a percepção dos estudantes universitários sobre os motivos que os levam a procrastinar suas tarefas acadêmicas; b) obter a frequência de procrastinação em quatro tarefas acadêmicas específicas; c) identificar possíveis relações entre as tarefas acadêmicas mais procrastinadas e os motivos associados a esse comportamento; d) identificar possíveis diferenças entre homens e mulheres em relação aos motivos da procrastinação acadêmica; e) obter evidências de validade para o instrumento através de procedimentos de análise fatorial e de correlações com depressão, impulsividade e conscienciosidade.

\section{Método}

\section{Participantes}

Os alunos de graduação que responderam aos instrumentos de maneira completa compuseram a amostra final que conteve 604 sujeitos. Amédia de idade foi de 25,15 anos $(D P=7,98)$, sendo $73,7 \%$ do sexo feminino. A quase totalidade dos participantes (97,8\%) era proveniente do estado do Rio Grande do Sul, sendo que $89,2 \%$ estudavam em instituição pública. Cerca de $65 \%$ dos alunos indicaram que seu rendimento acadêmico (em termos de notas obtidas) é superior a $80 \%$. Os cursos mais bem representados na amostra foram: Letras (24,3\%), Sociologia (14,1\%), História $(9,8 \%)$ e Psicologia (9,1\%). Em torno de $84 \%$ dos participantes afirmaram estar no curso que mais gostariam de estar nesse momento, cerca de $57 \%$ estavam cursando pela primeira vez um curso de nível superior e $43 \%$ dos estudantes já haviam iniciado outro curso.

\section{Instrumentos}

Procrastinação acadêmica. $\mathrm{O}$ instrumento de procrastinação construído foi chamado de "Escala de Motivos da Procrastinação Acadêmica" (EMPA), contendo duas seções. A primeira seção apresenta quatro itens com tarefas acadêmicas, para as quais o respondente deve marcar a frequência com que procrastina as mesmas, além de indicar a tarefa que mais procrastina. As tarefas são: fazer trabalhos que têm prazo de entrega (trabalhos); estudar para provas (provas); fazer exercícios ou leituras recomendadas da semana (exercícios); estudar os conteúdos de aula da semana ou rever anotações (estudo). A frequência é pontuada através de uma escala Likert de 5 pontos $(1=$ Nunca $; 2=$ Quase nunca $; 3=$ Às vezes; $4=$ Quase sempre; 5 = Sempre). Na segunda seção, nove itens (a versão final contou com apenas oito itens, como explicado adiante) apresentam possíveis motivos que levam o respondente a procrastinar a tarefa indicada como a mais procrastinada ("O quanto cada um dos motivos abaixo reflete os motivos pelos quais você procrastina nessa tarefa?"). A escala de respostas utilizada é do tipo Likert, variando de 1 a 5 ( $1=N a ̃ o$ reflete de modo algum; 3 = Reflete mais ou menos; $5=$ Reflete perfeitamente). O instrumento foi planejado de modo que escores mais altos nesse conjunto inicial de nove itens refletiriam uma maior propensão a experienciar motivos para procrastinar. 
Depressão. A Center for Epidemiologic StudiesDepression Scale (CES-D) é um instrumento internacional utilizado para avaliar depressão, que já foi adaptado e validado no Brasil (Silveira \& Jorge, 2000; Hauck-Filho \& Teixeira, 2011). Embora o instrumento proporcione também uma avaliação de fatores específicos da depressão (afetos negativos, afetos positivos, relações interpessoais e sintomas somáticos), é possível um escore geral de depressão ao somar todos os itens (após inverter itens sobre afetos positivos), estratégia mais interessante ao presente estudo. A consistência interna para o total dos itens do instrumento foi 0,90 pelo coeficiente alpha de Cronbach e 0,91 pelo coeficiente lambda6 de Guttman.

Conscienciosidade. Foram utilizados os cinco adjetivos relativos ao fator conscienciosidade do instrumento Marcadores reduzidos para avaliação da personalidade (Hauck-Filho, Machado, Teixeira, \& Bandeira, 2012). Um escore calculado a partir da soma dos adjetivos que compõem o fator reflete o nível de conscienciosidade (organização, responsabilidade e auto-diligência) de um indivíduo. A consistência interna dos cinco adjetivos foi de 0,81 pelo coeficiente alpha de Cronbach e de 0,80 pelo lambda6 de Guttman.

Impulsividade. Utilizou-se a subescala Fun Seeking das Escalas Behavioral Inhibition System e Behavioral Activation System (BIS-BAS; Carver \& White, 1994) com base na literatura que sugere que o sistema neurobiológico motivacional de aproximação está na base de comportamentos impulsivos e de busca de sensações (Carver \& White, 1994). Um escore composto pela soma dos itens desse fator reflete a propensão de um indivíduo ao envolvimento em comportamentos de busca por gratificações imediatas, possivelmente em detrimento do planejamento de metas em médio e longo prazo. O coeficiente alpha foi de 0,58 e o coeficiente lambda6 de Guttman foi de 0,51 .

\section{Procedimentos}

Este estudo seguiu todos os padrões éticos de pesquisa incluindo a Resolução 466/12 do Conselho Nacional de Saúde e o projeto foi aprovado pelo Comitê de Ética em Pesquisa do Instituto de Psicologia da Universidade Federal do Rio Grande do Sul sob o protocolo 89.727. Não existiram conflitos de interesse. Os potenciais participantes foram contatados através de convite eletrônico enviado à rede de contatos dos pesquisadores e a coordenações de alguns cursos superiores escolhidos aleatoriamente. Solicitou-se que cada participante convidasse outros universitários. Os instrumentos ficaram disponíveis para acesso através do link da pesquisa em tempo integral por 40 dias no site Survey Monkey.

\section{Construção do instrumento}

Para construção da EMPA, utilizou-se a Pure Procrastination Scale (Steel, 2010) e a Procrastination Assessment Scale for Students (PASS) (Solomon \& Rothblum, 1984) como diretrizes para a quantidade de itens e a âncora de respostas dos mesmos respectivamente. De maneira semelhante, a escolha de cada uma das tarefas acadêmicas incluídas na EMPA foi resultado de uma revisão das tarefas mais citadas em estudos e instrumentos sobre esse tema, tendo-se levado em consideração também se estas tarefas descritas na literatura eram usuais no contexto acadêmico brasileiro.

A seção sobre motivos da procrastinação da Procrastination Assessment Scale for Students inspirou a elaboração dos itens da EMPA em termos de conteúdo. No entanto, a PASS busca avaliar os motivos que levaram os estudantes a procrastinar um trabalho escrito final de uma disciplina da faculdade. Já a EMPA avalia os motivos que levaram o estudante a procrastinar a tarefa que costuma ser a mais procrastinada entre as quatro opções dadas (fazer trabalhos, estudar para provas, estudo diário e fazer exercícios ou leituras). Entre os 26 itens dessa seção da PASS, avaliou-se que muitos possuíam conteúdos semelhantes que refletiam, em essência, um mesmo motivo para procrastinar. Assim sendo, esses principais temas/motivos foram agrupados e, a partir daí, itens novos foram gerados com base nos seguintes temas: ansiedade, preguiça, baixa autoeficácia, perfeccionismo, falta de energia, tarefa difícil, falta de tempo e trabalhar sob pressão.

Todos os itens elaborados foram submetidos, previamente, à análise independente de três juízes que fizeram sugestões de ajustes após avaliarem qualitativamente a pertinência dos mesmos como indicadores do conteúdo do instrumento. Além disso, os juízes verificaram aspectos relativos à adequação técnica da escrita dos itens e a facilidade de compreensão/interpretação dos mesmos pelos universitários que participariam do estudo. Após uma reunião entre os juízes para discussão das sugestões propostas por cada um, implementaram-se as alterações que foram consenso no grupo.

\section{Análise dos dados}

As respostas quanto à frequência de procrastinação de cada uma das tarefas acadêmicas foram analisadas em termos de média, desvio padrão e frequência. A respeito de qual tarefa acadêmica era a mais procrastinada pelo 
respondente, a frequência de respostas foi analisada em termos percentuais. Em seguida, analisou-se a média geral e específica dos motivos associados à tarefa escolhida como a mais procrastinada pelo respondente.

Realizou-se uma análise fatorial exploratória dos itens referentes aos motivos da procrastinação com a finalidade de investigar a sua estrutura latente e de correlacionar os fatores encontrados às variáveis depressão, conscienciosidade e impulsividade. Em virtude da natureza ordinal dos dados, a análise fatorial foi conduzida utilizando o estimador Unweighted Least Squares (ULS) a partir de uma matriz de correlações policóricas, estratégia ideal para itens em escala Likert (Asún, Rdz-Navarro, \& Alvarado, 2015). O método de retenção de fatores foi a análise paralela com permutação dos dados empíricos (Timmerman \& Lorenzo-Seva, 2011). A fidedignidade da escala foi avaliada por meio de análises de consistência interna (alpha de Cronbach e lambda6 de Guttman). Por fim, por meio de testes de comparação de médias ( $t$ de Student), foram exploradas eventuais diferenças entre os sexos em função dos fatores relativos aos motivos da procrastinação. As análises foram conduzidas com o pacote psych (Revelle, 2014) do programa R e com o programa Factor 10.3.01 (Lorenzo-Seva \& Ferrando, 2013).

\section{Resultados}

Os resultados indicaram que $82,6 \%$ dos participantes $(n=499)$ afirmaram que procrastinam tarefas acadêmicas com alguma frequência. Em três das quatro tarefas acadêmicas, mais de $50 \%$ dos participantes procrastinadores indicaram procrastinar, no mínimo, "quase sempre". A Tabela 1 apresenta a média, o desvio padrão e o percentual indicados pelos participantes em relação a cada uma das tarefas.

Entre as quatro tarefas acadêmicas referidas, solicitou-se que os estudantes assinalassem somente aquela em que mais procrastinavam. A tarefa estudo foi indicada por $40,3 \%$; em seguida, a tarefa trabalho foi assinalada por $25,1 \%$ dos estudantes, enquanto que $23,6 \%$ afirmaram que procrastinam mais a tarefa exercícios. Os demais estudantes (11\%) indicaram a tarefa estudar para provas como a mais procrastinada.

Tendo em vista somente a tarefa acadêmica em que mais procrastinavam, os estudantes indicaram o quanto cada um dos motivos apresentados refletia os motivos pelos quais eles procrastinavam aquela tarefa. A média geral (ou seja, a média calculada independente de qual fosse a tarefa mais procrastinada) e as médias específicas de cada motivo em relação às tarefas acadêmicas são apresentadas na Tabela 2 .

TABELA 1

Média, desvio-padrão e frequência em percentual para cada tarefa acadêmica $(n=499)$

\begin{tabular}{lccccc}
\hline Tarefa & $M$ & $D P$ & $\begin{array}{c}\text { Nunca ou } \\
\text { Quase nunca } \\
\%(n)\end{array}$ & $\begin{array}{c}\text { As vezes } \\
\%(n)\end{array}$ & $\begin{array}{c}\text { Sempre ou } \\
\text { Quase sempre } \\
\%(n)\end{array}$ \\
\hline Estudo & 3,67 & 1,13 & $16,2(81)$ & $24,8(124)$ & $59,0(294)$ \\
Trabalhos & 3,45 & 1,26 & $24,2(121)$ & $22,2(111)$ & $53,5(267)$ \\
Exercícios & 3,38 & 1,00 & $17,8(89)$ & $39,1(195)$ & $43,1(215)$ \\
Provas & 3,36 & 1,26 & $27,5(137)$ & $21,2(106)$ & $51,3(256)$ \\
\hline
\end{tabular}

Nota: As médias podem variar de 1 a 5 , conforme a chave de respostas utilizada (onde $1=$ Nunca; $2=$ Quase nunca; $3=$ Às vezes; $4=$ Quase sempre; $5=$ Sempre).

TABELA 2

Média geral e específica dos motivos para procrastinar a tarefa acadêmica elencada como a mais procrastinada $(\mathrm{n}=499)$

\begin{tabular}{|c|c|c|c|c|c|}
\hline Motivos / Média & Geral & Provas & Estudo & Trabalhos & Exercícios \\
\hline 2. Tenho preguiça de fazer a tarefa. & 3,57 & 3,55 & 3,63 & 3,44 & 3,62 \\
\hline 5. Sinto-me cansado e sem energia para começar a tarefa. & 3,53 & 3,36 & 3,55 & 3,49 & 3,61 \\
\hline 8. Falta-me tempo para fazer a tarefa. & 3,24 & 3,18 & 3,21 & 2,95 & 3,19 \\
\hline 7. Eu realmente não gosto de realizar a tarefa. & 3,14 & 3,18 & 3,22 & 2,95 & 3,19 \\
\hline 4. Eu espero fazer a tarefa tão bem feita que tenho dificuldade em começar. & 2,77 & 2,67 & 2,52 & 3,10 & 2,87 \\
\hline 6. Acho elevado o grau de dificuldade da tarefa. & 2,46 & 2,56 & 2,39 & 2,48 & 2,49 \\
\hline $\begin{array}{l}\text { 1. Fazer a tarefa me deixa ansioso(a), pois fico com medo de não conseguir } \\
\text { fazer direito ou atingir um bom resultado. }\end{array}$ & 2,11 & 2,07 & 1,77 & 2,61 & 2,18 \\
\hline 9. Eu me motivo mais para realizar a tarefa se estou sob pressão. & 2,05 & 1,84 & 1,93 & 2,26 & 2,12 \\
\hline 3. Eu não me sinto capaz de fazer sozinho a tarefa. & 1,72 & 1,49 & 1,68 & 1,82 & 1,78 \\
\hline
\end{tabular}


Na sequência, conduziu-se uma análise fatorial exploratória dos motivos para procrastinar. Apenas dois autovalores empíricos superaram a variância explicada pela média dos autovalores encontrados na análise paralela, e apenas um autovalor empírico superou a variância explicada pelo percentil 95 da distribuição dos autovalores paralelos. Em outras palavras, a análise sugeriu um ou dois fatores como explicativos de variância não atribuível, exclusivamente, ao acaso. Decidiu-se em favor da solução de dois fatores, explicativa de 52,73\% da variância nos dados.

A seguir, foram estimados os parâmetros da análise fatorial exploratória com dois fatores. Inicialmente, observou-se que somente o item 7 ("Eu realmente não gosto de realizar a tarefa") não atingiu uma carga acima de 0,30 em nenhum dos fatores, motivo pelo qual foi deletado e uma nova análise foi conduzida. A TABELA 3 apresenta os resultados da análise final com oito itens, incluindo cargas fatoriais, comunalidades $\left(h^{2}\right)$ e índices de consistência interna. Os fatores se mostraram correlacionados, sendo $r=0,20, p<0,05$.

Verificou-se que os itens com cargas salientes no Fator 1 avaliam aspectos relacionados à preguiça, percepção de dificuldade associada à tarefa, cansaço, falta de energia e de tempo para realizar a tarefa. Esse conjunto de elementos sugere que há algum nível de esgotamento do indivíduo em relação ao quanto consegue se envolver com suas tarefas, pois ele avalia o nível de vigor para isso. Afinal, por um lado, as tarefas são percebidas como difíceis e, pelo outro lado, o indivíduo sente-se desanimado e desinteressado em realizá-las. Portanto, esse fator parece estar associado à baixa motivação acadêmica dos universitários e foi denominado como Procrastinação-desmotivação. Seu alpha de Cronbach foi 0,61 .
Os itens com cargas salientes no Fator 2 possuem conteúdos relacionados a preocupações com a realização da tarefa. Os itens desse fator tratam sobre sentimento de incapacidade para realizar a tarefa sozinho, medo de não conseguir fazer direito a tarefa e dificuldade para começar a tarefa em função de o estudante ter uma alta expectativa em relação ao quão bem ele espera fazê-la. Além disso, esse fator inclui um item indicando que o indivíduo percebe a necessidade de um estado de tensão - produzido pelo prazo - para que haja uma efetiva mobilização para a tarefa. Assim, esse fator representa um modo de agir mais inseguro, preocupado e perfeccionista, o que possivelmente leva a uma postergação da tarefa até o momento em que não é mais possível adiá-la, quando então o indivíduo sente-se forçado a executá-la. Em função disso, esse fator foi denominado Procrastinação-ansiedade. $\mathrm{O}$ alpha de Cronbach observado para a escala composta pelos itens do Fator 2 foi 0,76.

Sobre os resultados das correlações entre os fatores encontrados e as demais variáveis, ambos os fatores estiveram positiva e significativamente relacionados a escores da escala CES-D, como visto na Tabela 4. Isso indica que afetos negativos são possíveis constituintes da motivação procrastinatória. Não obstante, essas correlações foram de baixa magnitude. Além disso, ambos os fatores da Escala de Motivos da Procrastinação Acadêmica estiveram negativamente relacionados à dimensão conscienciosidade, ainda que os tamanhos de efeito tenham sido pequenos, e tenha havido significância estatística apenas para o Fator 1 (Procrastinaçãodesmotivação). Já entre os dois fatores encontrados e a dimensão Behavioral Activation System - Fun Seeking (BAS-FS) que avalia a impulsividade, as correlações foram de baixa magnitude e não foram significativas.

TABELA 3

Resultados da análise fatorial dos itens sobre motivos da procrastinação acadêmica

\begin{tabular}{|c|c|c|c|}
\hline Item & $F 1$ & $F 2$ & $h^{2}$ \\
\hline $\begin{array}{l}\text { 1. Fazer a tarefa me deixa ansioso(a), pois fico com medo de não conseguir fazer direito ou atingir um } \\
\text { bom resultado. }\end{array}$ & $-0,18$ & 0,84 & 0,69 \\
\hline 2. Tenho preguiça de fazer a tarefa. & 0,74 & $-0,09$ & 0,52 \\
\hline 3. Eu não me sinto capaz de fazer sozinho a tarefa. & 0,06 & $\mathbf{0 , 7 3}$ & 0,56 \\
\hline 4. Eu espero fazer a tarefa tão bem feita que tenho dificuldade em começar. & $-0,11$ & $\mathbf{0 , 5 0}$ & 0,24 \\
\hline 5. Sinto-me cansado e sem energia para começar a tarefa. & 0,47 & $-0,03$ & 0,22 \\
\hline 6. Acho elevado o grau de dificuldade da tarefa. & 0,59 & $-0,01$ & 0,34 \\
\hline 8. Falta-me tempo para fazer a tarefa. & 0,35 & 0,04 & 0,13 \\
\hline 9. Eu me motivo mais para realizar a tarefa se estou sob pressão. & 0,02 & 0,63 & 0,40 \\
\hline Fidedignidade dos fatores rotacionados & 0,68 & 0,82 & \\
\hline Alpha & 0,61 & 0,76 & \\
\hline Lambda6 & 0,56 & 0,74 & \\
\hline
\end{tabular}

F1 = Procrastinação-desmotivação; F2 = Procrastinação-ansiedade; $h^{2}=$ comunalidade . 
TABELA 4

Correlações bivariadas

\begin{tabular}{cccc}
\hline Fatores & $C E S-D$ & $C$ & $B A S-F S$ \\
\hline F1 & $0,18^{*}$ & $-0,18^{*}$ & 0,07 \\
F2 & $0,22^{*}$ & $-0,12$ & $-0,06$ \\
\hline
\end{tabular}

$* p<0,05$.

CES-D = Center for Epidemiologic Studies-Depression Scale;

$\mathrm{C}=$ Conscienciosidade; BAS-FS = Behavioral Activation System-Fun Seeking

Por fim, foram exploradas diferenças entre os sexos no que diz respeito aos fatores encontrados. Ainda que o sexo feminino tenha apresentado escores médios mais altos do que o sexo masculino em ambos os fatores $\left(\mathrm{F} 1_{\text {mulheres }}=3,23, \mathrm{~F} 1_{\text {homens }}=3,12\right.$, $\left.\mathrm{F} 2_{\text {mulheres }}=2,20, \mathrm{~F} 2_{\text {homens }}=2,06\right)$, as diferenças não foram estatisticamente significativas. $\mathrm{O}$ tamanho de efeito para as diferenças foi de pequena magnitude em ambas as comparações de médias, sendo $d=0,13$ para o Fator 1, e $d=0,15$ para o Fator 2. Os achados, portanto, sugerem um padrão bastante similar entre os sexos no que diz respeito à frequência dos diferentes motivos da procrastinação acadêmica.

\section{Discussão}

O presente trabalho apresentou as etapas de construção dos itens da Escala de Motivos da Procrastinação Acadêmica, análises psicométricas dos oito itens escolhidos para compor o instrumento, e ainda relações entre os fatores da escala e variáveis externas. Um conjunto inicial de nove itens de autorrelato foi, após a análise semântica por juízes, respondido por uma amostra de estudantes universitários. A análise fatorial sugeriu que oito desses nove itens são indicadores adequados de dois fatores latentes que representam tendências à procrastinação acadêmica: Procrastinaçãodesmotivação e Procrastinação-ansiedade. Enquanto o primeiro fator endereça aspectos de adiamento da tarefa em função de falta de iniciativa, interesse, cansaço e preguiça, o segundo fator está mais relacionado à procrastinação motivada por afetos negativos, como preocupação e expectativas muito altas acerca do próprio desempenho na tarefa procrastinada. A fidedignidade das escalas resultantes foi modesta $(0,61$ e 0,76 , respectivamente), mas bastante razoável tendo em vista o reduzido número de itens de cada escala apenas quatro.

As associações entre os fatores do instrumento e as variáveis externas coletadas também se mostraram interpretáveis de acordo com a literatura. Especificamente, as correlações positivas com depressão e negativas com conscienciosidade seguiram o padrão verificado em uma meta-análise de 216 estudos (Steel, 2007). Assim, apesar da reduzida magnitude, esse padrão é consistente com a tendência de associação descrita na literatura, fornecendo evidências de validade para a EMPA.

Medo do fracasso, baixos níveis de energia, baixa motivação, baixa autoconfiança e baixa autoeficácia comumente são fatores associados à depressão e que podem, em conjunto ou isoladamente, aumentar o nível de dificuldade que o estudante tem para realizar uma tarefa acadêmica ou alterar a percepção do mesmo a respeito do quão aversiva é essa tarefa para ele (Steel, 2007). Ademais, esses achados lançam luz aos possíveis processos cognitivos representados pelos fatores do instrumento, ressaltando que ambos os fatores parecem envolver uma falta de planejamento/organização combinada a afetos negativos que podem contribuir para o surgimento do comportamento procrastinatório nesse contexto acadêmico.

Entre os cinco grandes fatores de personalidade, a conscienciosidade tem sido apontada como aquele que possui a relação mais forte com a procrastinação, explicando boa parte da variabilidade dos escores obtidos em medidas de procrastinação (Watson, 2001). Uma vez que a conscienciosidade é composta por facetas tais como competência, ordem, deliberação, busca de realização e autodisciplina é compreensível que baixos níveis de conscienciosidade estejam associados a níveis mais altos de procrastinação (Watson, 2001). Afinal, sujeito mais autodisciplinados possuem uma tendência menor de adiar suas tarefas acadêmicas desnecessariamente como ocorre na procrastinação, pois talvez valorizem mais finalizar uma tarefa importante ainda que não estivessem muito dispostos a realizá-la. Esse padrão de correlação negativa, além de fazer sentido teoricamente, condiz tanto com o que foi encontrado nesse estudo e como com o que vem sendo descrito na literatura (Watson, 2001).

Curiosamente, a escala de BAS-fun seeking, que avalia aspectos da impulsividade, não se mostrou relacionada a nenhum dos dois fatores. Isso, ao contrário de ser um demérito ao instrumento, apenas sugere que o aspecto central da procrastinação acadêmica talvez esteja mais relacionado à conscienciosidade e aos afetos negativos. No entanto, uma expansão do número de itens e maior detalhamento de seu conteúdo (abrangendo diversos aspectos da impulsividade vistos a partir do próprio comportamento procrastinatório) pode esclarecer essas possíveis relações e revelar um terceiro fator subjacente à EMPA. Afinal, a impulsividade tem se correlacionado com a procrastinação em diversos estudos, indicando que pode ocorrer um abandono da tarefa acadêmica em prol de uma gratificação mais 
imediata sem que haja uma prévia consideração a respeito das consequências dessa decisão (Steel, 2007).

Em relação à comparação entre os sexos, não foi identificada uma diferença estatisticamente significativa nos fatores do instrumento. $\mathrm{O}$ tamanho de efeito $(d)$ observado indica que a pequena diferença talvez seja de pouca importância de um ponto de vista prático ou teórico. Como discutido em outros estudos, o comportamento de procrastinar não apresenta diferenças substanciais quando se compara homens e mulheres e, quando diferenças são observadas, elas tendem a ser pequenas ou moderadas (Enumo \& Kerbauy, 1999; Solomon \& Rothblum, 1984, Steel, 2007). Entretanto, com relação aos motivos da procrastinação, poucos estudos (Ozer, Demir, \& Ferrari, 2009; Sharma \& Kaur, 2011) exploraram possíveis diferenças entre os sexos. Portanto, os resultados ainda não são conclusivos.

Outros achados do presente estudo também chamam a atenção, uma vez que contribuem no sentido de mapear o comportamento procrastinatório de estudantes universitários brasileiros. Neste estudo, aproximadamente $83 \%$ dos participantes afirmaram que procrastinam tarefas acadêmicas com alguma frequência, dado semelhante ao encontrado por Sampaio e Bariani (2011) em seu estudo com universitários brasileiros. Entre as tarefas acadêmicas, a mais procrastinada foi o estudo diário. A realização dessa tarefa costuma exigir um maior nível de autocontrole e de autorregulação dos estudantes, já que os mesmos devem estabelecer suas próprias metas de estudo, o que poderia aumentar a ocorrência de procrastinação, uma vez que é considerada uma falha no processo de autorregulação da aprendizagem (Sampaio, 2011).

$\mathrm{O}$ rendimento acadêmico superior a $80 \%$ e a alta prevalência de procrastinação na amostra podem gerar a impressão de que a procrastinação não é um comportamento prejudicial. Chu e Choi (2005) argumentam sobre a possibilidade de existir um tipo de procrastinador que escolhe procrastinar, pois prefere trabalhar sob pressão. Esse tipo de procrastinador foi denominado "procrastinador ativo" e possui características de autoeficácia, estilo de coping, controle do tempo e resultados acadêmicos semelhantes aos dos estudantes que não procrastinam suas tarefas acadêmicas (Chu \& Choi, 2005). Por outro lado, alguns procrastinadores utilizam mecanismos de compensação para evitar prejuízos acadêmicos tais como ingerir bebidas estimulantes para ficar estudando até o último instante possível para uma prova ou pedir ajuda a colegas para finalizar um trabalho. Além disso, existem evidências de que procrastinação acadêmica e comportamentos de trapaça na universidade são variáveis correlacionadas positiva e moderadamente
(Merce, Concepcion, M. del Mar, \& Ramon, 2012) o que reforça a ideia de que os estudantes procuram evitar prejuízos no seu rendimento acadêmico depois de terem procrastinado.

Um dos objetivos do presente estudo foi identificar os motivos mais alegados pelos estudantes para procrastinarem cada uma de suas tarefas acadêmicas. De modo geral, o motivo mais relatado foi preguiça de fazer a tarefa. Esse motivo obteve a maior média geral entre as tarefas e também foi o principal motivo alegado para a procrastinação das tarefas estudo, provas e exercícios. A preguiça pode ser entendida como reflexo de uma motivação baixa para a realização da atividade. Quando a tarefa é considerada como aborrecedora, pouco relevante ou pouco interessante, fica difícil transformar a intenção de realizar a tarefa em ação de fato (Conceição, 2011). Já em relação à tarefa trabalhos, o principal motivo indicado foi cansaço e falta de energia para começar a tarefa. Certa exaustão costuma ser o motivo mais citado pelos estudantes entre as possíveis alterações de estado físico e mental que poderiam explicar esse comportamento (Grunschel, Patrzek, \& Fries, 2013a).

Logo após a preguiça e o cansaço, a falta de tempo surge enquanto o terceiro motivo mais alegado para a procrastinação de tarefas acadêmicas de modo geral. Essa falta de tempo pode estar relacionada a fatores tais como dificuldades de planejar e de priorizar tarefas, sobrecarga de trabalho e percepção distorcida do tempo necessário à realização da tarefa (Conceição, 2011). Portanto, a falta de tempo pode ocorrer em função de uma má gestão do tempo (Conceição, 2011).

Não gostar de realizar a tarefa surgiu como o quarto motivo mais alegado para procrastinação acadêmica. Uma tarefa que alguém não gosta de realizar pode ser descrita como uma tarefa desagradável, desinteressante, entediante ou muito difícil. Essa falta de satisfação para realizar uma atividade tem sido apontada por cerca de $25 \%$ dos universitários como um motivo para que procrastinem (Sampaio \& Bariani, 2011). Nesses casos, a procrastinação pode ser entendida como uma resposta comportamental de evitação (Schouwenburg, 2004; Steel, 2007), pois espera-se que um indivíduo procrastine mais ao perceber uma tarefa como sendo punitiva, aversiva, ou não suficientemente recompensadora (Conceição, 2011). Outro motivo indicado foi a ansiedade de se fazer a tarefa em função do medo de não conseguir fazê-la direito ou de não atingir um bom resultado. Isso pode indicar que os estudantes apresentam expectativas irrealistas e perfeccionismo que podem levar a extensas revisões do trabalho que dificultam sua finalização (Conceição, 2011; Steel, 2007). 
A tentativa de identificar perfis ou tipos de procrastinadores também tem sido um foco das pesquisas (Ferrari, Johnson, \& McCown, 1995; Grunschel, Patrzek, \& Fries, 2013b, Lay, 1986). Mais recentemente, Grunschel, Patrzek e Fries (2013b) conduziram uma análise latente do perfil de 554 estudantes universitários e sugeriram dois possíveis perfis de procrastinadores acadêmicos: aqueles mais ansiosos/preocupados e aqueles que estão descontentes com seus estudos (Grunschel, Patrzek, \& Fries, 2013b). Logo, os fatores Procrastinação-ansiedade e Procrastinação-desmotivação do presente estudo também são compatíveis com a explicação de que a procrastinação é um comportamento de evitação que ocorre, principalmente, em função do medo e do desprazer associados à realização da tarefa. Nesse sentido, a ansiedade pode estar relacionada a dificuldades de planejamento e tomada de decisões que influenciariam tanto no surgimento como na manutenção do comportamento procrastinatório - podendo a ansiedade ser um motivo e/ou uma consequência da procrastinação. Por enquanto, sabe-se que a ansiedade está correlacionada, positivamente, com a procrastinação e, negativamente, com a motivação intrínseca (Chu \& Choi, 2005). Todas essas possíveis relações não foram exploradas neste estudo - o que representa uma limitação do mesmo e uma sugestão para a realização de estudos posteriores.

Futuros estudos podem explorar se os fatores Procrastinação-ansiedade e Procrastinação-desmotivação representam tipos diferentes de procrastinadores acadêmicos ou se são dimensões que podem explicar, em conjunto, o comportamento da maioria dos sujeitos. Sabe-se que fatores implicam diferenças quantitativas entre os indivíduos, enquanto classes latentes caracterizam diferenças qualitativas - pois os indivíduos são similares entre si dentro das classes. Portanto, há um contraste entre os resultados de Grunschel, Patrzek e Fries (2013b) e os apresentados nesse estudo. É possível que não existam "tipos" de procrastinadores, mas sim duas dimensões latentes comuns a todos os indivíduos que, aos serem correlacionadas, admitem múltiplas configurações de escores. Para isso, futuros estudos podem verificar se os dois fatores relacionados à motivação para procrastinação acadêmica estão correlacionados - o que indicaria uma diferença quantitativa entre os sujeitos.

Entre as limitações deste estudo encontra-se a natureza da amostra que foi não probabilística e o fato de ter incluído, majoritariamente, estudantes de apenas um estado da federação. Portanto, outras pesquisas que ampliem o perfil das amostras (diferentes cursos, regiões do país e características sociodemográficas) são fundamentais para que tais achados possam ser generalizados. Porém, destaca-se que a quantidade de participantes foi adequada para realizar as análises estatísticas empregadas - o que faz pensar que os resultados obtidos possam ser replicados em outras amostras. O fato de a coleta ter sido realizada através da Internet também pode ser considerada uma limitação, na medida em que inexiste qualquer tipo de controle sobre a veracidade das informações obtidas, incluindo o próprio perfil dos participantes. Além disso, deve-se considerar que, como em qualquer instrumento de autorrelato, não é o comportamento real que está sendo avaliado, e sim a percepção do respondente sobre o seu comportamento.

Outra limitação dessa pesquisa é o potencial processo de autosseleção da amostra. É possível que aqueles participantes que optaram por colaborar com o estudo procrastinem menos, uma vez que responderam ao instrumento dentro do prazo da coleta de dados. Por outro lado, alguns estudantes podem ter preenchido os instrumentos enquanto procrastinavam a realização de alguma tarefa acadêmica. Logo, não se sabe qual foi o exato impacto do processo de autosseleção na composição da amostra. Por outro lado, houve um predomínio de estudantes de instituições públicas de ensino e de cursos de ciências humanas que deve ser considerado antes de se generalizar os resultados desse estudo para outras populações. Afinal, estudantes de ciências exatas podem apresentar outros resultados em função de diferenças relacionadas às características de suas tarefas, nível de exigência, entre outros fatores.

\section{Considerações finais}

Além de explorar a prevalência da procrastinação acadêmica, o presente estudo apresentou a construção da Escala de Motivos da Procrastinação Acadêmica que poderá ser útil para a realização de pesquisas brasileiras sobre os motivos da procrastinação acadêmica. Pela natureza breve do instrumento, pode ser possível sua utilização em estudos de larga escala sobre habilidades não cognitivas dos estudantes brasileiros. A partir dos resultados dessas pesquisas, será possível comparar estudos brasileiros com outros internacionais, demonstrando o potencial da EMPA de servir como ferramenta para se avançar na construção do conhecimento sobre o tema da procrastinação acadêmica em diversos contextos.

Em sala de aula, o instrumento pode ser utilizado tanto para registro do comportamento dos estudantes como para levantamento de possíveis motivos do comportamento procrastinatório. A partir disso, podem ser desenvolvidas atividades específicas para um determinado grupo de alunos com o objetivo de 
promover a autorregulação da aprendizagem naquelas atividades ou situações onde se encontram as maiores dificuldades dos estudantes. Assim, a orientação e a elaboração de programas de prevenção relacionados ao tema podem ser feitos de forma mais ágil e focada nas reais necessidades dos estudantes. Nessas intervenções, o instrumento pode ser útil enquanto um dispositivo de promoção da reflexão dos estudantes sobre seu comportamento a partir de uma discussão coletiva ou da análise individual das respostas dadas.

Além de ser útil a pedagogos, orientadores educacionais e psicólogos na intervenção direta com os estudantes, os dados obtidos com a EMPA em nível institucional podem ser norteadores da formulação de novas estratégias de ensino com base na análise dos resultados gerais feita pelos próprios professores e demais envolvidos no ensino universitário. Por fim, o instrumento pode ser útil para comparar aspectos do comportamento procrastinatório antes e depois de uma intervenção, desde que haja um intervalo de tempo razoável entre cada aplicação da EMPA de modo que os estudantes não recordem com precisão a resposta dada em um primeiro momento de coleta de dados.

\section{Referências}

Asún, R. A., Rdz-Navarro, K., \& Alvarado, J. M. (2015). Developing multidimensional Likert Scales using item factor analysis: The case of four-point items. Sociological Methods \& Research, 45(1). https://doi. org $/ 10.1177 / 0049124114566716$

Burka, J. B., \& Yuen, L. M. (1983). Procrastination: Why you do it, what to do about it. Reading, MA: Addison-Wesley.

Carver, C. S. \& White, T. L. (1994). Behavioral inhibition, behavioral activation and affective responses to impending reward and punishment: The BIS-BAS scales. Journal of Personality and Social Psychology, 67, 319-333. https:// doi.org/10.1037/0022-3514.67.2.319

Cerino, E. S. (2014). Relationships between academic motivation, self-efficacy, and academic procrastination. Psi Chi Journal of Psychological Research, 19(4), 156-163. https://doi.org/10.24839/2164-8204.JN19.4.156

Conceição, J. P. O. (2011). Personalidade e procrastinação em estudantes universitários. Dissertação de Mestrado, Universidade Lusófona de Humanidades e Tecnologias, Lisboa: Portugal.

Costa, M. (2007). Procrastinação, auto-regulação e gênero. Dissertação de Mestrado, Universidade do Minho, Guimarães: Portugal.

Chu, A. H. C. \& Choi, J. N. (2005). Rethinking procrastination: positive effects of "active" procrastination behavior on attitudes and performance. The Journal of Social Psychology, 145, 245-264. https://doi.org/10.3200/SOCP.145.3.245264

Effert, B. R. \& Ferrari, J. R. (1989). Decisional procrastination: Examining personality correlates. Journal of Social Behavior and Personality, 4, 151-161.

Grunschel, C., Patrzek, J., \& Fries, S. (2013a). Exploring reasons and consequences of academic procrastination: An interview study. European Journal of Psychology of Education, 28(3), 841-861. https://doi.org/10.1007/s10212-0120143-4

Grunschel, C., Patrzek, J., \& Fries, S. (2013b). Exploring different types of academic delayers: A latent profile analysis. Learning and Individual Differences, 23, 225-233. https://doi.org/10.1016/j.lindif.2012.09.014

Enumo, S. \& Kerbauy, R. (1999). Procrastinação: Descrição de comportamentos de estudantes e transeuntes de uma capital brasileira. Revista Brasileira de Terapia Comportamental e Cognitiva, 2, 125-135.

Hamasaki, E. I. M. \& Kerbauy, R. R. (2001). Será o comportamento de procrastinar um problema de saúde? Revista Brasileira de Terapia Congitiva Comportamental, 3, 35-40.

Hauck-Filho, N. \& Teixeira, M. A. P. (2011). A estrutura fatorial da Escala CES-D em estudantes universitários brasileiros. Avaliação Psicológica, 10, 91-97.

Hauck-Filho, N., Machado, W. L., Teixeira, M. A. P., \& Bandeira, D. (2012). Marcadores reduzidos para a avaliação da personalidade em adolescentes. Psico-USF, 17(2), 253-261. https://doi.org/10.1590/S1413-82712012000200009

Hollender, M. H. (1965). Perfectionism. Comprehensive Psychiatry, 6, 94-103. https://doi.org/10.1016/S0010440X(65)80016-5

Kerbauy, R. (2001). Análise funcional da preguiça e procrastinação. In R. C. Wielenska (Org.). Sobre cognição e comportamento: questionando e ampliando a teoria e as intervenções clínicas e em outros contentos (pp. 63-69). Santo André, SP: ESETec Editores Associados.

Klingsieck, K. B. (2013). Procrastination: When good things don't come to those who wait. European Psychologist, 18(1), 24-34. https://doi.org/10.1027/1016-9040/a000138

Lay, C. H. (1986). At last my research article on procrastination. Joumal of Research in Personality, 20, 474-495. https:// doi.org/10.1016/0092-6566(86)90127-3 
Lorenzo-Seva, U. \& Ferrando, P. J. (2013). FACTOR 9.2: A comprehensive program for fitting exploratory and semiconfirmatory factor analysis and IRT models. Applied Psychological Measurement, 37(6), 497-498. https://doi. org/10.1177/0146621613487794

McCown, W., Johnson, J., \& Petzel, T. (1989). Procrastination, a principal components analysis. Personality and Individual Differences, 10, 197-202. https://doi.org/10.1016/0191-8869(89)90204-3

Merce, C., Concepcion, G., M. del Mar, B., \& Ramon, C. (2012). Procrastination and Cheating from Secondary School to University. Electronic Journal of Research in Educational Psychology, 10(2), 737-754.

O'Brien, W. K. (2002). Applying the transtheoretical model to academic procrastination. Tese de doutorado, University of Houston, Houston.

Ozer, B. U., Demir, A., \& Ferrari, J.R. (2009). Exploring academic procrastination among Turkish students: Possible gender differences in prevalence and reasons. The Journal of Social Psychology, 149, 241-257. https://doi.org/10.3200/ SOCP.149.2.241-257

Pychyl, T. A., Lee, J. M., Thibodeau, R., \& Blunt, A. (2000). Five days of emotion: An experience sampling study of undergraduate student procrastination. Journal of Social Behavior and Personality, 15, 239-254.

Revelle, W. (2014). Psych: Procedures for personality and psychological research. R package version 1.4.3. CRAN Project. Retirado de: <http://cran.r-project.org/web/packages/psych/psych.pdf>. Acessado em: 11 dez. 2015.

Sampaio, R. K. N. (2011). Procrastinação acadêmica e a autorregulação da aprendizagem em estudantes universitários. Dissertação de Mestrado. Universidade Estadual de Campinas, Campinas, SP.

Sampaio, R. K. N.; \& Bariani, I. C. D. (2011). Procrastinação Acadêmica: Um estudo exploratório. Estudos Interdisciplinares em Psicologia, 2(2), 242-262. https://doi.org/10.5433/2236-6407.2011v2n2p242

Schouwenburg, H. (1995). Academic Procrastination: Theoretical notions, measurement and research. In J. Ferrari, J. Johnson, \& W. McCown, Procrastination and task avoidance: Theory, research and practice (pp. 71- 950). New York: Plenum Press. https://doi.org/10.1007/978-1-4899-0227-6_4

Sharma, M. \& Kaur, G. (2011). Gender differences in procrastination and academic stress among adolescents. Indian Journal of Social Science Researches, 8(2), 122-127.

Silveira, D. X. \& Jorge, M. R. (2000). Escala de Rastreamento Populacional para Depressão CES-D em populações clínicas e não clínicas de adolescentes e adultos jovens. In C. Gorestain, L. H. S. G. Andrade, \& A. W. Zuarde (Orgs.). Escalas de avaliação clínica em psiquiatria e farmacologia (pp. 125-135). São Paulo: Lemos.

Solomon, L. \& Rothblum, E. (1984). Academic procrastination: Frequency and cognitive-behavioural correlates. Journal of Counselling Psychology, 31(4), 503-509. https://doi.org/10.1037/0022-0167.31.4.503

Schouwenburg, H. (2004). Trait procrastination in academic settings: An overview of students who engage in task delays. In H. Schouwenburg, C. Lay, T. Pylchyl, \& J. Ferrari (Eds.). Counselling the procrastinator in academic settings (pp. 3-18). Washington: American Psychological Association.

Steel, P. (2007). The nature of procrastination: A meta-analytic and theoretical review of quintessential self-regulatory failure. Psychological Bulletin, 133, 65-94. https://doi.org/10.1037/0033-2909.133.1.65

Steel, P. (2010). Arousal, avoidant and decisional procrastinators: Do they exist? Personality and Individual Differences, 48, 926-934.

Timmerman, M. E. \& Lorenzo-Seva, U. (2011). Dimensionality assessment of ordered polytomous items with parallel analysis. Psychological Methods, 16(2), 209-220. https://doi.org/10.1037/a0023353

Watson, D. C. (2001). Procrastination and the five-factor model: a facet level analysis. Personality and Individual Differences, 30(1), 149-158. https://doi.org/10.1016/S0191-8869(00)00019-2

Zimmerman, B. J. \& Schunk, D. H. (Eds.). (2001). Self-regulated learning and academic achievement: Theoretical perspectives. Mahwah, NJ: Erlbaum. 


\section{ANEXO}

\section{ESCALA DE MOTIVOS DA PROCRASTINAÇÃO ACADÊMICA (VERSÃO INICIAL)}

Este questionário avalia a procrastinação acadêmica. A procrastinação acadêmica é entendida como a tendência que um estudante pode apresentar em deixar para fazer depois ou para o último minuto tarefas acadêmicas importantes, tais como manter os estudos em dia, fazer exercícios ou leituras recomendadas, organizar anotações de aula, preparar-se para provas e fazer trabalhos que precisam ser entregues. Alguns alunos procrastinam mais, outros menos, e outros ainda não procrastinam. Queremos conhecer um pouco mais o quanto isso ocorre com os estudantes. Para isso pedimos que você responda as questões e itens abaixo.

- Se procrastina, com que frequência você procrastina nas tarefas acadêmicas abaixo?

\begin{tabular}{|c|c|c|c|c|}
\hline 1 & 2 & 3 & 4 & 5 \\
\hline Nunca & Quase nunca & Às vezes & Quase sempre & Sempre \\
\hline
\end{tabular}

\begin{tabular}{|c|c|c|c|c|c|c|}
\hline & TAREFA & \multicolumn{5}{|c|}{ RESPOSTA } \\
\hline 1 & Estudar os conteúdos de aula da semana ou rever anotações da semana & 1 & 2 & 3 & 4 & 5 \\
\hline 2 & Fazer exercícios ou leituras recomendadas da semana & 1 & 2 & 3 & 4 & 5 \\
\hline 3 & Estudar para provas & 1 & 2 & 3 & 4 & 5 \\
\hline 4 & Fazer trabalhos que têm prazo de entrega & 1 & 2 & 3 & 4 & 5 \\
\hline
\end{tabular}

- Se você procrastina, em qual tipo de tarefa (entre as acima) isso mais ocorre?

$$
1(\text { ) } 2(\text { ) } 3(\text { ) } 4(\text { ) }
$$

- O quanto cada um dos motivos abaixo reflete os motivos pelos quais você procrastina nessa tarefa?

\begin{tabular}{|c|c|c|c|c|}
\hline 1 & 2 & 3 & 4 & 5 \\
\hline Não reflete de modo algum & - & Reflete mais ou menos & - & Reflete perfeitamente \\
\hline
\end{tabular}

\begin{tabular}{|c|c|c|c|c|c|c|}
\hline & MOTIVO & \multicolumn{5}{|c|}{ RESPOSTA } \\
\hline 1 & $\begin{array}{l}\text { Fazer a tarefa me deixa ansioso(a) pois fico com medo de não conseguir fazer direito } \\
\text { ou atingir bom resultado. }\end{array}$ & 1 & 2 & 3 & 4 & 5 \\
\hline 2 & Tenho preguiça de fazer a tarefa. & 1 & 2 & 3 & 4 & 5 \\
\hline 3 & Eu não me sinto capaz de fazer sozinho a tarefa. & 1 & 2 & 3 & 4 & 5 \\
\hline 4 & Eu espero fazer a tarefa tão bem feita que tenho dificuldade em começar. & 1 & 2 & 3 & 4 & 5 \\
\hline 5 & Sinto-me cansado e sem energia para começar a tarefa. & 1 & 2 & 3 & 4 & 5 \\
\hline 6 & Acho elevado o grau de dificuldade da tarefa. & 1 & 2 & 3 & 4 & 5 \\
\hline 7 & Eu realmente não gosto de realizar a tarefa. & 1 & 2 & 3 & 4 & 5 \\
\hline 8 & Me falta tempo para fazer a tarefa. & 1 & 2 & 3 & 4 & 5 \\
\hline 9 & Eu me motivo mais para realizar a tarefa se estou sob pressão. & 1 & 2 & 3 & 4 & 5 \\
\hline
\end{tabular}

\footnotetext{
Autores:

Nelson Hauck Filho - Doutor, Universidade São Francisco.

Endereço para correspondência:

Gabriela Ballardin Geara

Av. Protásio Alves, 1469, apto 08 - Bairro Rio Branco

90410-001, Porto Alegre, RS, Brasil

<gearagabi@gmail.com>
}

Gabriela Ballardin Geara - Doutoranda, Universidade Federal do Rio Grande do Sul.

Marco Antonio Pereira Teixeira - Doutor, Universidade Federal do Rio Grande do Sul.

Recebido: 26.07.2016

Aceito: 04.11.2016 